\title{
A NOTE ON UMBILICS OF A CLOSED SURFACE
}

\author{
MINORU KURITA
}

In this paper we investigate indices of umbilics of a closed surface in the euclidean space. Most part of the discussion is concerned with a symmetric tensor field of degree 2, or rather a direction field, on a Riemannian manifold of dimension 2 .

Let $M$ be a compact orientable Riemannian manifold of class 2 and $X$ be a direction field (a field of non-oriented tangents) of class 2 on $M$. We can take in a coordinate neighborhood $U$ rectangular frames $e_{1}, e_{2}$ according to which the line element $d s$ is given by

$$
d s^{2}=\omega_{1}^{2}+\omega_{2}^{2} .
$$

Then we can take such $\omega_{12}$ that

$$
d \omega_{1}=\omega_{2} \wedge \omega_{21}, \quad d \omega_{2}=\omega_{1} \wedge \omega_{12}, \quad \omega_{12}=-\omega_{21},
$$

and then

$$
d \omega_{12}=-K \omega_{1} \wedge \omega_{2},
$$

where $K$ is the gaussian curvature. For a direction field $X$ with some singular points we can take such a neighborhood $V$ of any point $p \in U$ that in $V$ the direction field is given by $\left(l_{1}, l_{2}\right)$ with respect to the rectangular frame, where $l_{1}, l_{2}$ are functions of class 2 . We put

$$
D l_{1}=d l_{1}+l_{2} \omega_{21}, \quad D l_{2}=d l_{2}+l_{1} \omega_{12}
$$

and also

$$
\tau=\frac{l_{1} D l_{2}-l_{2} D l_{1}}{l_{1}^{2}+l_{2}^{2}}
$$

Then we get

$$
\tau=\frac{l_{1} d l_{2}-l_{2} d l_{1}}{l_{1}^{2}+l_{2}^{2}}+\omega_{12}=d\left(\tan ^{-1} \frac{l_{2}}{l_{1}^{-}}\right)+\omega_{12} .
$$

Hence $\tau$ is a form which depends on the ratio $l_{1}: l_{2}$ and not on $l_{1}, l_{2}$. $\tau$ is also independent with a choice of rectangular frames on $U$, because $D l_{1}, D l_{2}$ are

Received February 4, 1959. 
covariant differential of a vector $\left(l_{1}, l_{2}\right)$ defined on $V$. Thus $\tau$ defined on a neighborhood $V$ of each point determines a 1 -form on $M$ which is related with our direction field $X$. We have by (6), (3)

$$
d \tau=d \omega_{12}=-K d S
$$

where $d S$ means an element of area on $M$.

Let the singular points of the direction field on $M$ be $p_{1}, \ldots, p_{k}$ and $D_{1}$, $\ldots, D_{k}$ be their neighborhoods with boundaries $C_{1}, \ldots C_{k}$. By integrating (7) on the domain $M-\sum_{i=1}^{k} D_{i}$ we get for suitable orientations of $C_{i}$

$$
\int_{M} K d S-\sum_{i=1}^{k} \int_{D_{i}} K d S=\sum_{i=1}^{k} \int_{C_{i}} \tau .
$$

When we contract $D_{i}$ to the point $p_{i}$, we get

$$
\lim \int_{c_{i}} \tau=n_{i} \pi
$$

with an integer $n_{i}$, because we have by (6)

$$
\int_{C_{i}} \tau=\int_{C_{i}} d\left(\tan ^{-1} \frac{l_{2}}{l_{1}}\right)+\int_{C_{i}} \omega_{12}=n_{i} \pi+\int_{C_{i}} \omega_{12}, \quad \lim \int_{C_{i}} \omega_{12}=0 .
$$

If we contract each domain $D_{i}$ to $p_{i}$, we get from (8)

$$
\int_{M} K d S=\sum_{i=1}^{k} n_{i} \pi
$$

Now, denoting by $\%$ Euler-Poincare's characteristic of $M$ we have

$$
\int_{M} K d S=2 \pi \chi
$$

and (10) reduces to

$$
2 \chi=\sum_{i=1}^{k} n_{i}
$$

We call $n_{i}$ an index of a singular point $p_{i}$ of the direction field. This can also be interpreted as follows. We develope a curve $C_{i}$ on the euclidean plane. If we denote by $l$ a vector developed on the euclidean plane corresponding to $\left(l_{1}, l_{2}\right), D l_{1}$ and $D l_{2}$ are components of $d l$ and we get

$$
\int_{c_{i}} \tau=\int_{c_{i}} \frac{|[l, d l]|}{|l|^{2}}=\int_{c_{i}} d \varphi
$$

where $[l, d l]$ means a vector product of $l$ and $d l$, and $d \varphi$ an infinitesimal angle 
of rotation of $l$. Thus $n_{i} \pi$ is a limiting value of an angle of rotation of our direction field when developed on the euclidean plane along $C_{i}$.

Thus we get the following lemma.

Lemma. Let $M$ be a 2-dimensional compact orientable Riemannian manifold and $\chi$ be its Euler-Poincare's characteristic. For a direction field with a finite number of sigular points of finite indices $n_{i}(i=1, \ldots, k)$ we have

$$
2 \%=\sum_{i=1}^{k} n_{i}
$$

This is already known as is shown in [2] p. 207, but the proof above stated will be of use later in our discussion.

Now we take up a closed surface $M$ of class 2 in the euclidean space of dimension 3. By taking tagent rectangular frames the line element on $M$ can be given as in (1) and the 2nd fundamental form by

$$
a_{11} \omega_{1}^{2}+2 a_{12} \omega_{1} \omega_{2}+a_{22} \omega_{2}^{2} .
$$

Thus a symmetric tensor $\left(a_{i j}\right)$ is defined on $M$. If we denote by $a_{i j k}$ covariant derivatives of $a_{i j}$ we have

$$
a_{i j k}=a_{i k j} .
$$

Umbilics of $M$ are points for which the eigenvalues of $\left(a_{i j}\right)$ are equal, and the directions of eigenvectors are principal directions when the eigenvalues are different. We take on $M$ a direction field defined by directions of eigenvectors corresponding to a greater one of eigenvalues of the tensor $\left(a_{i j}\right)$. This field is of class 2 , because the greater eigenvalue is an analytic function of $a_{i j}$ in the neighborhood of points which are not umbilic, and the singular points of the direction field are umbilics. We define an index of an umbilic by the index of the direction field. Then by virtue of lemma we get

THEOREM 1. If a closed surface in the euclidean space has a finite number of umbilics whose indices are $n_{1}, \ldots, n_{k}$, we have

$$
2 \chi=\sum_{i=1}^{k} n_{i}
$$

where $\%$ denotes Euler-Poincare's characteristic of the surface.

As a corollary 
THEOREM 2. A closed surface in the euclidean space with non-vanishing Euler-Poincare's characteristic has an umbilic.

Theorem 1 was proved by $\mathrm{W}$. Blaschke for an analytic surface homeomorphic to a sphere (cf. [1] p. 288).

Next we return to $X$. We take a domain $D$ on $M$ and let $p_{1}, \ldots, p_{h}$ be singular points of the direction field in $D$ and $D_{1}, \ldots, D_{h}$ be domains in $D$, each containing $p_{1}, \ldots, p_{h}$ respectively, and $C_{1}, \ldots, C_{h}$ be their boundaries. Then by integrating (7) for a domain $D-\sum_{i=1}^{h} D_{i}$ and contracting each $D_{i}$ to $p_{i}$, we get

$$
\int_{D} K d S=\sum_{i=1}^{h} n_{i} \pi-\int_{C} \tau
$$

where $n_{i}$ are indices of $p_{i}$, and $C$ is a boundary of $D$.

We take up again the case of umbilic and assume that the domain above stated is a polygon of $p$-sides whose laterals are segments of lines of curvature and inner angles are all a right angle. Along the boundary $C$ of the domain $D$ we take such rectangular frames that one of the basic vectors $e_{1}, e_{2}$ is tangent to the lateral (lines of curvature) and we always have the relation $l_{2}=0$. And if we denote by $k$ a geodesic curvature of lines of curvature and by $d s$ an arcelement along them, we get by (6)

$$
\int_{C} \tau=\int_{C} d\left(\tan ^{-1} \frac{l_{2}}{l_{1}}\right)+\int_{C} \omega_{12}=\int_{C} \omega_{1 z}=\int_{C} k d s .
$$

If $D$ is simply connected, we get by virtue of Gauss-Bonnet's theorem

$$
\int_{D} K d S=2 \pi-\int_{C} k d s-p \frac{\pi}{2}
$$

By (15), (16), (17) we get

$$
\sum_{i=1}^{h} n_{i}=2-\frac{p}{2} .
$$

Thus we get

THEOREM 3. If $D$ is a simply connected domain on a surface in the euclidean space and the boundary of $D$ are segments of lines of curvature with inner angles all equal to a right angle, the sum of indices of umbilics contained in $D$ is equal to $2-\frac{p}{2}$. 
If $p=0$ and an umbilic is contained in $D$, an index of the umbilic is equal to 2 , as is in the case of umbilics on an ellipsoid of unequal axes, and if $p=2$ and an umbilic is contained in $D$, an index of the umbilic is equal to 1 , as is in the case of umbilics on an ellipsoid of rotation which is not a sphere.

A relation (14) has not been utilized in our investigation. The author wants to know what properties can be deduced about umbilics from the relation,

\section{LITERATURE}

[1] W. Blaschke, Vorlesungen über Differentialgeometrie III, 1929.

[2] N. Steenrod, The topology of fibre bundles, 1951.

\section{Mathematical Institute}

Nagoya University 